\title{
Editorial
}

\section{0 years of change and progress}

\author{
P. C. Hewlett
}

It seems appropriate after 60 years to look back at what Magazine of Concrete Research (MCR) has achieved as a technical publication dedicated to concrete and put it alongside the development in concrete as a material as well.

The first issue of MCR appeared in January 1949 and was 44 pages long. The board then comprised three people with the editorial being anonymous and the first of the three papers in that issue dealt with reinforced concrete beams in shear - we are still publishing such papers today!

There were technical papers, research notes, apparatus and methods, and current literature. Correspondence and discussion about the papers was encouraged although no author's affiliation was given. The messages were clearly stated namely

(a) a plea for scientific research into concrete

(b) co-ordination of research to minimise duplication

(c) results of the research should be useful

(d) identify principle problems and they should be a priority

(e) encourage early disclosure.

Notwithstanding many changes to concrete and the research community that underwrites it $M C R$ has retained many of the initial motivations and attitudes without compromising the presentational style and quality.

The board in turn has evolved responding to these changes and now comprises in total 12 members with three overseas corresponding members. Each issue now consists of approximately 80 pages and we receive about 250 submissions each year. All submissions are carefully reviewed by at least two board members.

Concrete is global as are the papers we receive. Every effort is made to publicise accepted papers promptly including pre-publication disclosure on the internet.

Over the intervening period there have been many innovations in design, performance expectations and materials relating to concrete. More recently, climate change and sustainability issues have focused on concrete's 'carbon footprint' as well as whole-life performance, including the benefit provided by its thermal mass in a warming climate. Notwithstanding these issues concrete remains the most widely used building fabric and in many situations the material of first choice.

The presentational and written style of $M C R$ is still similar to the original format although the apparatus and methods and current literature sections are infrequent nowadays. There has been over the intervening period welcome input of papers from the Far East, Malaysia and India, reflecting global research activities into concrete.

A review of volume 30 (March 1978) showed that it also consisted of 48 pages and the editorial was concerned about papers given at conferences that might not otherwise have been published. Such concerns have been addressed over the intervening period with a growing emphasis on matters material as well as structural.

With hindsight a number of items are worth mentioning.

(a) We do not receive much correspondence about published papers these days. Such disclosure should be encouraged since it mobilises current thinking and action.

(b) Much research into concrete does not transfer into adopted practice both on site and in the design 
office - why not? Site-used concrete and laboratory-made concrete can be very different. We need both materials' data to represent engineering reality.

(c) Reproducible and reliable information from real sites and constructions is needed to feedback into realistic service design lives.

(d) Much research is aimed at providing answers to perceived questions. More emphasis should be placed on asking the right questions to begin with.

(e) Performance of concrete buildings in service and over time particularly applies to remedial techniques. The emphasis should be on proven performance rather than prescription.
( $f$ ) Research papers on environmental performance/ sustainability should be encouraged to deal with the fundamentals of this important subject.

Having said all of this, over the last 60 years concrete and buildings based on it have advanced and will continue to do so. The global demand for concrete continues to rise and it is to be hoped that $M C R$ will also continue to act as a global communicator and play its role in assisting beneficial change based on sound and relevant research and development. 